\title{
Routes and regulation of NADPH production in steroidogenic mitochondria
}

\author{
Israel Hanukoglu, and Revital Rapoport \\ The Research Institute, The College of Judea and Samaria, Ariel 44820, and \\ Institute of Endocrinology, Sourasky-Tel Aviv Medical Center, Tel-Aviv, Israel.
}

Fax: 972-3-9640796.

\begin{abstract}
The first and rate-limiting step of steroidogenesis is catalyzed by the mitochondrial cholesterol side chain cleavage system that is dependent on NADPH. The pathways of NADPH generation in steroidogenic mitochondria include three major routes catalyzed by: 1. NADP-linked malic enzyme, 2. NADP-linked isocitrate dehydrogenase, and 3 . nicotinamide nucleotide transhydrogenase. The main route may differ among cell types and across species. Generally operation of alternative routes, with different substrates is not excluded. The oxidation of NADPH by the mitochondrial P450 systems is not tightly coupled with substrate metabolism, as these systems can reduce $\mathrm{O}_{2}$ by a single electron to produce harmful superoxide radical. To minimize such futile NADPH oxidation, NADPH generation may be regulated by two types of mechanisms: 1 . Feedback mechanisms that maintain the ratio of NADPH/NADP+ at a steady-state level by enhancing the rate of NADPH production to keep up with its rate of oxidation, e.g., allosteric regulation of enzymes involved in NADPH production. 2. Hormonal signals that enhance the level of NADPH production in coordination with steroidogenesis. One major hypothesis with experimental evidence is that stimulation of mitochondrial $\mathrm{NAD}(\mathrm{P}) \mathrm{H}$ synthesis is mediated by $\mathrm{Ca}^{++}$as a second messenger of tropic factors. Tropic stimulation of cells increases the levels of $\mathrm{Ca}^{++}$in the cytosol and then in the mitochondrial matrix, and the rise in $\mathrm{Ca}^{++}$activates enzymes involved in $\mathrm{NAD}(\mathrm{P}) \mathrm{H}$ synthesis. These regulatory mechanisms most probably operate in concert adjusted to the steroidogenic activity of the cell.
\end{abstract}




\section{INTRODUCTION}

The tropic hormones of steroidogenic tissues can stimulate maximal steroidogenic response within a few minutes. This rapid process involves the mobilization of cholesterol from intracytoplasmic vesicles and its transfer into the mitochondria where cholesterol is converted into pregnenolone by the side chain cleavage system $(1,2)$. This conversion and most of the subsequent steps of steroid hormone biosynthesis are monooxygenation reactions (hydroxylations) catalyzed by cytochrome P450 type enzymes (3). These reactions depend on molecular oxygen and electrons donated by NADPH with the following stoichiometry:

$$
\mathrm{R}-\mathrm{H}+\mathrm{NADPH}+\mathrm{H}^{+}+\mathrm{O}_{2} \rightarrow \mathrm{R}-\mathrm{OH}+\mathrm{NADP}^{+}+\mathrm{H}_{2} \mathrm{O}
$$

The electrons are transferred from NADPH to $\mathrm{P} 450$ by an electron transfer system. The NADPH specificity of these systems derives from the cofactor specificity of the electron transfer proteins. The mitochondrial P450 systems are located on the matrix side of the inner membrane, and include an NADPH specific adrenodoxin reductase and adrenodoxin $(3,4)$. The microsomal systems depend on a single NADPH specific $\mathrm{P} 450$ reductase. Some microsomal P450s may receive the second electron from NADH through cytochrome $b_{5}$ reductase and cytochrome $b_{5}$ (5).

This review summarizes studies on the pathways and regulation of NADPH synthesis and consumption by the mitochondrial P450 systems. Early research on the mechanism of acute stimulation of steroidogenesis examined the pathways of NADPH supply (6). Yet, as the research trends shifted to other areas, this subject was left incompletely understood. The studies reviewed provide evidence that NADPH supply to the mitochondrial P450 systems is regulated and coupled to steroidogenesis, yet, the mechanisms of this coupling remain to be elucidated.

\section{ROUTES OF NADPH BIOSYNTHESIS IN MITOCHONDRIA}

Studies during the 1950's and later showed that Krebs cycle intermediates such as succinate, isocitrate and $\alpha$-ketoglutarate could support steroid hydroxylation in mitochondria (6-9). The actions of these metabolites were shown to be dependent on their stimulation of $\mathrm{NADP}^{+}$reduction to produce NADPH $(6,10)$.

The reactions of the Krebs cycle take place in the mitochondrial matrix bounded by the highly impermeable inner mitochondrial membrane. Yet, substrates of the cycle can be transported into the mitochondria by specific carriers, such as the pyruvate carrier, and di- and tricarboxylate carriers (11). Thus, the effects of substrates on intra-mitochondrial reactions can be studied by their addition into purified intact isolated mitochondria. 
A major source of NADPH in the cell is the cytosolic pentose phosphate pathway. However, NADPH cannot be transported into mitochondria (12). Exogenously added NADPH can be used in mitochondrial steroid hydroxylation only if the mitochondria are permeabilized by high concentrations of $\mathrm{Ca}^{++}$or other treatments $(8,12)$.

The major routes of NADPH production in the mitochondria of steroidogenic cells are depicted in Fig. 1. This scheme includes three routes of NADPH synthesis catalyzed by: 1. NADP linked malic enzyme, 2. NADP linked isocitrate dehydrogenase, and 3 . nicotinamide nucleotide transhydrogenase. Although, many studies on this subject have pointed out a specific major route, they have generally not excluded operation of other routes, with different intermediates.

Because of the cyclic nature of the Krebs cycle, the addition of a specific substrate can stimulate NADPH production at more than one site. Thus, the operation of a route was generally shown by testing the ability of a specific substrate to support steroid hydroxylation in the presence of inhibitors of specific routes. The interpretation of these studies is further complicated by the use of inhibitors that may affect more than one reaction, the secondary effects of the substrates, the method of preparation of the mitochondria, the degree of coupling of oxidative phosphorylation and other factors (13).

The involvement of malic enzyme in NADPH synthesis in bovine adrenocortical mitochondria was indicated by the observation that pyruvate was formed during fumarate supported reactions, concomitant with the reduction of NADP+ (14, 15). Malic enzyme is a 65 kdalton protein (16) that catalyzes the following reaction:

$$
\text { L-malate }+\mathrm{NADP}^{+} \rightleftarrows \text { pyruvate }+\mathrm{CO}_{2}+\mathrm{NADPH}+\mathrm{H}^{+}
$$

At the initial characterization of this enzymatic activity it was suggested to be the major source of NADPH for steroid hydroxylation in adrenal cortex mitochondria (14). However, many studies in diverse systems showed the existence of alternative pathways, and questioned the centrality of the role of this enzyme in NADPH synthesis (for review see ref. 13). Inorganic phosphate $\left(\mathrm{P}_{\mathrm{i}}\right)$ was shown to stimulate malate uptake into mitochondria and malate dependent pyridine nucleotide reduction, presumably via malic enzyme (17).

Isocitrate dehydrogenase catalyzes decarboxylation of isocitrate to form $\alpha$ ketoglutarate according to the following reaction:

isocitrate $+\mathrm{NAD}(\mathrm{P})^{+}+\mathrm{H}^{+} \rightleftarrows \alpha$-ketoglutarate $+\mathrm{CO}_{2}+\mathrm{NAD}(\mathrm{P}) \mathrm{H}$

In the mitochondria there are two forms of isocitrate dehydrogenase, an NAD specific form and an NADP specific form. The NADP specific enzyme is considered as one source of NADPH for steroid hydroxylation based on the observations that isocitrate can stimulate steroid hydroxylation without being 


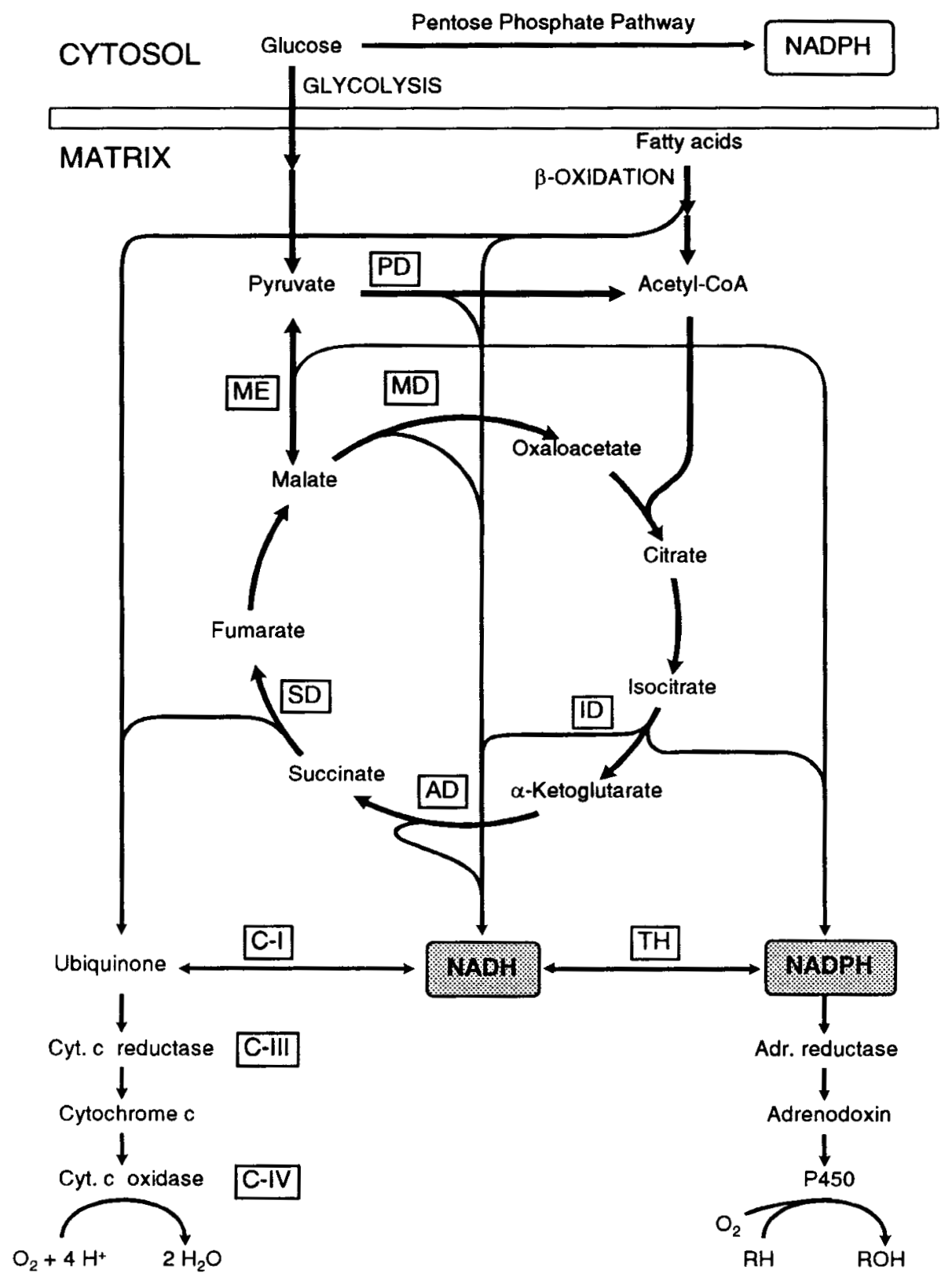

FIGURE 1

Routes of NADPH synthesis in the mitochondria of steroidogenic cells. Abbreviations: $\mathrm{AD}, \alpha$-ketoglutarate dehydrogenase; ID, isocitrate dehydrogenase; $\mathrm{MD}$, malate dehydrogenase; $\mathrm{ME}$, malic enzyme; $\mathrm{PD}$, pyruvate dehydrogenase; SD, succinate dehydrogenase; TH, transhydrogenase; C-I, C-III, and C-IV, complexes of the electron transport chain of oxidative phosphorylation system. 
completely inhibited by respiratory inhibitors (18). The utilization of this pathway apparently varies between cell types and mitochondrial preparations as isocitrate supported steroid hydroxylation can be inhibited to different degrees in various systems $(18,19)$.

One of the more intensively studied routes of NADPH synthesis is transhydrogenation with $\mathrm{NADH}(13,20)$. NADH is generated during some reactions of the Krebs cycle and during fatty acid $\beta$-oxidation (Fig. 1). The nicotinamide nucleotide transhydrogenase catalyzes the interconversion of NADH and NADPH according to the reaction:

$$
\mathrm{NADH}+\mathrm{NADP}+\rightleftarrows \mathrm{NAD}^{+}+\mathrm{NADPH}
$$

The transhydrogenase purified from bovine heart is a single polypeptide of 120 kdalton that also functions as a proton pump in the inner mitochondrial membrane (20). In the forward direction NADPH synthesis is coupled with $\mathrm{H}^{+}$translocation from the cytoplasmic side into the matrix of the mitochondria, and the converse occurs for the reverse reaction. Thus, because of its coupling to the proton $\left(\mathrm{H}^{+}\right)$ electrochemical gradient across the inner membrane, the transhydrogenase is also linked to oxidative phosphorylation (20). In addition to this transhydrogenase, mitochondrial enzymes that have both NAD and NADP dependent isoforms, such as isocitrate dehydrogenase, may also function as a transhydrogenase without being coupled to the proton electrochemical gradient (20).

The involvement of transhydrogenases in NADPH production is indicated by several lines of evidence: 1. Addition of NADH into permeabilized mitochondria supports steroid hydroxylation, despite the NADPH specificity of the P450 systems (21). 2. Increased levels of NADH is correlated with enhanced steroid hydroxylation (21). 3. Stimulation of steroid hydroxylation by Krebs cycle intermediates can be inhibited by inhibitors of oxidative phosphorylation presumably by their inhibition of energy linked transhydrogenases $(9,19,21-23)$. 4. NADH generating fatty acid oxidation could support steroid hydroxylation while the Krebs cycle was inhibited (24).

Besides the routes noted above preliminary evidence has been reported for an ATP dependent reduction of $\mathrm{NADP}^{+}$by succinate in ovarian corpora lutea mitochondria (25).

A cursory comparison of the studies noted above shows that the major routes of NADPH generation may differ among steroidogenic cell types (e.g., adrenal cortex zones, and corpus luteum) and across species. Yet, this conclusion should be stated with the caveat that some differences might have been caused by different conditions of mitochondrial preparation and incubation.

As noted above, early studies suggested that several alternative routes of NADPH generation may be functional in steroidogenic mitochondria. Recent studies indicate that there may be subpopulations or compartments of cholesterol 
side chain cleavage systems with different metabolic environments and exposures to reducing equivalents $(9,26)$. Both isocitrate and succinate can support cholesterol side chain cleavage in rat adrenal mitochondria, at similar initial rates but different saturation levels $(9,26)$. The responses to these two substrates differed in additional parameters suggesting the presence of pools of cholesterol side chain cleavage activity with heterogeneous responsiveness $(9,26)$. The responses of P450scc and P450c11 activities to some substrates are synergistically enhanced by the presence of other substrates, thus showing complex interactions among the pathways (27).

\section{IS NADPH SYNTHESIS COREGULATED WITH STEROIDOGENESIS?}

Many of the studies summarized above showed that steroid metabolism by the mitochondrial P450s is stimulated rapidly upon addition of various intermediates in the pathways of NADPH synthesis. These results appear to indicate a lack of control in the utilization of NADPH and raise several questions regarding the supply and consumption of NADPH by the mitochondrial P450 systems: Is NADPH constantly available to the P450 systems even in absence of steroid substrates? Is NADPH synthesis coupled to steroid metabolism, and if so what are the mechanisms that co-regulate steroid substrate delivery and NADPH synthesis?

As in other metabolic systems, some control mechanisms would be expected to operate to minimize futile NADPH synthesis and utilization in steroidogenic mitochondria. A priori at least two control mechanisms can be hypothesized:

1. Coupling of NADPH oxidation by the mitochondrial P450 systems to steroid hydroxylation, i.e., NADPH is available to the P450 system freely, but its oxidation is dependent on the metabolism of a steroid substrate.

2. Coupling of NADPH synthesis to stimulation of steroidogenesis, i.e., the signals that stimulate steroidogenesis also stimulate NADPH synthesis.

Recent studies relevant to these hypotheses are summarized below.

\section{NADPH OXIDATION BY THE P450 SYSTEMS IS NOT TIGHTLY COUPLED TO STEROID METABOLISM}

Each steroid hydroxylation is dependent on two electron reduction of one molecule of $\mathrm{O}_{2}$. Besides this normal biochemical function, P450 systems can also reduce $\mathrm{O}_{2}$ by a single electron to produce harmful superoxide radical (28). This phenomenon has been termed as "uncoupling of electron transfer from hydroxylation" or as "leaky electron transfer".

To determine the degree of coupling in mitochondrial P450 systems we recently tested the ability of reconstituted P450scc and P450c11 systems to oxidize 
NADPH in the absence and presence of their respective steroid substrates $(29,30)$. Both P450scc and P450c11 systems were observed to oxidize NADPH in the absence of their substrates, transferring electrons to $\mathrm{O}_{2}$ and producing superoxide. This leakage of electrons appeared to flow mainly through the P450. Adrenodoxin reductase did not represent a significant source of electron leakage. The addition of the substrate cholesterol reduced the leakage from P450scc, leaving only about $10 \%$ of the electrons leaking from $\mathrm{P} 450 \mathrm{scc}$ to $\mathrm{O}_{2}$ for superoxide production, while the remainder was used in substrate hydroxylation to produce pregnenolone. $\mathrm{P} 450 \mathrm{c} 11$ was much more leaky than P450scc. During deoxycorticosterone metabolism by P450c11, more than 50\% of the electron flow from NADPH was consumed in oxygen radical formation. For both $\mathrm{P} 450$ systems the rates of steroid product and superoxide formation increased as NADPH concentration was increased (30).

The above findings and previous studies (see ref. 30) indicated that in mitochondrial P450 systems NADPH oxidation is not tightly coupled with substrate metabolism. Earlier studies in cultured adrenocortical cells had shown that in contrast to $\mathrm{P} 450 \mathrm{scc}, \mathrm{P} 450 \mathrm{c} 11$ can undergo rapid inactivation after addition of some "pseudosubstrate" steroids $(31,32)$. As this inactivation was prevented by antioxidants it was hypothesized that it represents a $\mathbf{P} 450$ generated oxygen radical mediated effect $(32,33)$. To test this hypothesis we determined the effects of the same series of steroids on oxygen radical formation in reconstituted systems with purified P450c11 (30). In these studies, the steroids with no adverse effect on P450c11 activity in cultured cells, also showed no stimulatory effect on electron leakage in the reconstituted $\mathrm{P} 450 \mathrm{c} 11$ system. Whereas, the steroids that enhanced P450c11 degradation in cells, also increased NADPH oxidation by the purified $\mathrm{P} 450 \mathrm{c} 11$ system. These stereospecificity correlations, and the finding that P450c11 is much more leaky than P450scc, provided strong evidence that the observations with purified enzymes do reflect electron transfer and leakage reactions in living cells (30).

A study that examined the redox state of adrenodoxin by ESR spectroscopy concluded that adrenodoxin remains fully reduced in hypophysectomized rat adrenals with or without ACTH treatment (34). These findings were interpreted to minimize the role of $\mathrm{ACTH}$ regulation of reducing equivalent supply (34). This interpretation requires a caveat that cellular metabolism could have changed within minutes after induction of anesthesia and prior to the removal of the adrenal. In reconstituted systems adrenodoxin has been invariably observed to be rapidly oxidized to generate superoxide radical (ref. 29 and refs. therein). The constant maintenance of adrenodoxin in reduced state would thus be expected to introduce what has been referred as an "electron gun" inside the mitochondria to produce harmful radicals. Yet, the possibility cannot be eliminated that inside the 
mitochondria the reduced forms of the P450 system proteins may remain stably reduced in absence of steroid metabolism. The high concentrations of antioxidants in steroidogenic cells have been hypothesized to serve as scavengers for the radicals produced by the $\mathrm{P} 450$ systems $(32,35,36)$.

\section{MECHANISMS OF REGULATION OF NADPH SYNTHESIS}

The observations summarized in the previous section indicate that if NADPH is constantly available to the mitochondrial P450 systems then NADPH would be oxidized in a futile cycle generating harmful oxy-radicals without coupling to steroid metabolism. If these results reflect the physiological situation in the cell, then it would be imperative to regulate the availability of NADPH to these enzymes to minimize their futile oxidation of NADPH.

The subject of regulation of NADPH synthesis raises many questions: Is the level of NADPH maintained at a steady-state while its rate of production is enhanced to keep up with its rate of oxidation? If so, what are the allosteric modulators and enzymes that regulate NADPH production? Is the level of NADPH increased in the mitochondria upon hormonal stimulation of steroidogenesis? If so, what are the second messengers and processes that effect this change? To simplify a discussion of these and other questions we shall consider two distinct but nonexclusive short-term mechanisms for regulation of NADPH levels in steroidogenic mitochondria:

1. Feedback mechanisms to maintain the ratio of NADPH/NADP+ at a near steady-state by enhancing the rate of NADPH production to keep up with its rate of oxidation. These mechanisms include regulation of enzyme activities by feedback loops of allosteric regulators. This type of mechanisms operate in the control of oxidative phosphorylation of ATP synthesis (37). A recent hypothesis suggested that transhydrogenase and NAD- and NADP-linked isocitrate dehydrogenases operate in cooperation to control the activity of the Krebs cycle (38), though the hypothesized direction of dehydrogenase function (NADPH $\rightarrow$ NADH) is opposite to that expected in steroidogenic mitochondria. By analogous mechanisms the synthesis of NADPH may be regulated by enzymes that are sensitive to redox levels of $\mathrm{NAD}(\mathrm{P})^{+}$, or accompanying changes in the matrix concentration of ions or substrates consumed during $\mathrm{NAD}(\mathrm{P}) \mathrm{H}$ generation. As noted above, one of the prominent enzymes in NADPH synthesis is transhydrogenase. The coupling of this enzyme to the electrochemical gradient and its regulation by ions and substrates indicate one control site for NADPH production (20).

2. Hormonal signals to regulate NADPH production in coordination with steroidogenesis. Some early studies suggested that tropic hormones regulate the Krebs cycle and NADPH production $(6,39)$. Besides tropic hormones, the steroid 
precursor for $11 \beta$-hydroxylation, deoxycorticosterone, was shown to enhance the Krebs cycle activity, while steroid end products, progesterone and corticosterone were observed to be inhibitory (39)

The strongest direct evidence for the hypothesis that tropic hormones enhance NADPH production was provided by recent studies in adrenal glomerulosa cells (40). Measurement of the intracellular concentration of $\mathrm{NAD}(\mathrm{P}) \mathrm{H}$ by fluorescence revealed that $\mathrm{NAD}(\mathrm{P}) \mathrm{H}$ level increases in parallel with aldosterone production in response to $\mathrm{K}^{+}$stimulation (40). The rise in $\mathrm{NAD}(\mathrm{P}) \mathrm{H}$ fluorescence was shown to be of mitochondrial origin as it could be duplicated by the application of amytal, an inhibitor of mitochondrial complex I. This also provided evidence that NADPH was probably generated from NADH by transhydrogenation (40).

One of the major signal transduction mechanisms in adrenal glomerulosa cells involves $\mathrm{Ca}^{++}(41)$. The $\mathrm{K}^{+}$induced rise in NAD(P)H level in adrenal glomerulosa cells is associated with an increase in the intracellular concentration of $\mathrm{Ca}^{++}$ $\left(\left[\mathrm{Ca}^{++}\right]_{\mathrm{i}}\right)$, suggesting that changes in $\left[\mathrm{Ca}^{++}\right]_{i}$ may regulate NAD $(\mathrm{P}) \mathrm{H}$ levels $(40)$. Among the many second messengers activated by tropic hormones (4), $\mathrm{Ca}^{++}$is one that can be transported from the cytosol across the mitochondrial inner membrane Thus, increased $\left[\mathrm{Ca}^{++}\right]_{\mathrm{i}}$ may also lead to an elevation of $\left[\mathrm{Ca}^{++}\right]$in the matrix (42). Studies in other types of cells and tissues indicated that enhanced $\mathrm{Ca}^{++}$levels in the matrix may stimulate some enzymes to enhance NAD(P)H synthesis (42).

In steroidogenic mitochondria feedback regulators and hormonal signals most probably operate in concert. The relative roles of these mechanisms probably depend on the steroidogenic activity of the cell.

In addition to short term mechanisms of enzyme activation and intermediate substrate metabolism, recent studies indicate that NADPH biosynthesis may also be regulated by long term mechanisms at the level of the expression of the enzymes in the pathways of $\mathrm{NAD}(\mathrm{P}) \mathrm{H}$ production. ACTH was shown to induce the expression of mitochondrial mRNAs encoding subunits of oxidative phosphorylation system enzymes, in parallel with the induction of steroidogenic enzyme genes in adrenocortical cells in culture $(43,44)$. In bovine corpora lutea a low but significant correlation was observed between mitochondrial P450 system enzymes and a cytochrome oxidase subunit (45). In mouse ovary the activities of isocitrate dehydrogenase and other enzymes of glucose metabolism vary in parallel with the steroidogenic capacity of the ovary (46). Yet, the expression of enzymes involved in energy production does not appear to be tropic hormone regulated in all types of steroidogenic cells (43, Lehoux et al., unpublished observations).

The questions raised above highlight major gaps in our understanding of regulation of reducing equivalent supply, and require further study of the regulation of NADPH regeneration for the mitochondrial P450 systems in steroidogenic cells. 


\section{REFERENCES}

1. Jefcoate CR, McNamara BC, Artemenko I, Yamazaki T. 1992 J Steroid Biochem Mol Biol 43:751-767.

2. Clark BJ, Stocco DM. 1995 Endocrine Res 21:---- (this issue).

3. Lambeth JD. 1990 Frontiers in Biotransformation 3:58-100.

4. Hanukoglu I. 1992 J Steroid Biochem Mol Biol 43:779-804.

5. Schenkman JB, Greim H. 1993 Cytochrome P450. (Handbook Expl. Pharmacol. vol. 105), Springer-Verlag, Berlin.

6. Haynes RC. 1975 In: Handbook of Physiology-Endocrinology vol. 6, Am. Physiol. Soc., Washington, D.C., pp. 69-76.

7. Brownie AC, Grant JK. 1954 Biochem J 57:255-263.

8. Cammer W, Estabrook RW. 1967 Arch Biochem Biophys 122:721-734.

9. McNamara BC, Jefcoate CR. 1990 Arch Biochem Biophys 283:464-471.

10. Grant JK, Brownie AC. 1955 Biochim Biophys Acta 18:433-434.

11. LaNoue KF, Schoolwerth AC. 1979 Ann Rev Biochem 48:871-922.

12. Hochberg RB, Ladany S, Welch M, Lieberman S. 1974 Biochemistry 13:19381945.

13. Rydstrom J, Hoek JB, Ernster L. 1976 In: Boyer PD (ed) The Enzymes. Third edition. Academic Press, New York. pp. 51-88.

14. Simpson ER, Cooper DY, Estabrook RW. 1969 Recent Progr Horm Res 25:523-562.

15. Simpson ER, Estabrook RW. 1969 Arch Biochem Biophys 129:384-395.

16. Magnuson MA, Morioka H, Tecce MF, Nikodem VM. 1986 J Biol Chem 261:1183-1186.

17. Sauer LA, Park R. 1973 Biochemistry 12:643-649.

18. Sauer LA, Mulrow PJ. 1969 Arch Biochem Biophys 134:486-496.

19. Uzgiris VI, McIntosh EN, Alonso C, Salhanick HA. 1971 Biochemistry 10:29162923.

20. Hoek JB, Rydstrom J. 1988 Biochem J 254:1-10.

21. Hall PF. 1972 Biochemistry 11:2891-2897.

22. Klein KO, Harding BW. 1970 Biochemistry 9:3653-3658.

23. Lin MT, Haksar A, Peron FG. 1974 Arch Biochem Biophys 164:429-439.

24. Harano Y, Kowal J. 1972 Arch Biochem Biophys 153:68-73.

25. Robinson J, Stevenson PM. 1972 FEBS Lett 23:327-331.

26. McNamara BC, Jefcoate CR. 1990 Mol Cell Endocrinol 73:123-134.

27. Yamazaki T, McNamara BC, Jefcoate CR. 1993 Mol Cell Endocrinol 95:1-11.

28. Archakov AI, Bachmanova GI. 1990 Cytochrome P-450 and active oxygen. Taylor \& Francis, Hants, U.K. 
29. Hanukoglu I, Rapoport R, Weiner L, Sklan D. 1993 Arch Biochem Biophys 305:489-498.

30. Rapoport R, Sklan D, Hanukoglu I. 1995 Arch Biochem Biophys, in press.

31. Hornsby PJ. 1980 J Biol Chem 255:4020-4027.

32. Hornsby PJ. 1989 Free Radicals Biol Med 6:103-115.

33. Hanukoglu I, Feuchtwanger R, Hanukoglu A. 1990 J Biol Chem 265:2060220608.

34. Williams-Smith DL, Simpson ER, Barlow SM, Morrison PJ. 1976 Biochim Biophys Acta 449:72-83.

35. Riley JCM, Behrman HR. 1991 Proc Soc Exp Biol Med 198:781-791.

36. Carlson JC, Wu XM, Sawada M. 1993 Free Radicals Biol Med 14:79-84.

37. Brown GC. 1992 Biochem J 284:1-13.

38. Sazanov LA, Jackson JB. 1994 FEBS Lett 344:109-116.

39. Lin MT, Haksar A, Peron FG. 1974 Biochem Biophys Res Commun 58:983-989.

40. Pralong WF, Hunyady L, Varnai, Wollheim CB, Spat A. 1992 Proc Natl Acad Sci USA 89:132-136.

41. Stern N, Yanagawa N, Saito F, Hori M, Natarajan R, Nadler J, Tuck M. 1993 Endocrinology 133:843-847.

42. McCormack JG, Denton RM. 1993 Biochem Soc Trans 21:793-799.

43. Raikhinstein M, Hanukoglu I. 1993 Proc Natl Acad Sci USA 90:10509-10513.

44. Raikhinstein M, Hanukoglu I. 1994 J Steroid Biochem Mol Biol 49:257-260.

45. Hanukoglu I, Hanukoglu Z. 1986 Eur J Biochem 157:27-31.

46. Chapman JC, Waterhouse TB, Michael SD. 1992 Biol Reprod 47:992-997. 